\title{
3.8 INTERSTELLAR SCATTERING AND THE PULSE FROM THE VELA PULSAR
}

\author{
M. M. KOMESAROFF \\ Division of Radiophysics, CSIRO, Sydney, Australia \\ P. A. HAMILTON \\ Department of Physics, University of Tasmania, Hobart, Australia \\ and \\ J. G. A BLES \\ Division of Radiophysics, CSIRO, Sydney, Australia
}

\begin{abstract}
Measurements of PSR 0833 - 45 were made at wavelengths of 21, 48, 73 and $100 \mathrm{~cm}$. Pulse broadening proportional to about the fourth power of the wavelength was found, consistent with multipath scattering in the interstellar medium. It is further concluded that the rotation measure was constant at $33.2 \mathrm{rad} \mathrm{m}^{-2}$ across this wavelength range, supporting the conclusion that the radiation originates in the vicinity of a magnetic pole.
\end{abstract}

\section{Introduction}

During February 1970 the pulsar PSR $0833-45$ was observed simultaneously at a number of wavelengths between 20 and $120 \mathrm{~cm}$ using the ANRAO 210-ft telescope at Parkes. The results indicated a dramatic broadening of the pulse at wavelengths longer than about $75 \mathrm{~cm}$ (Ables et al., 1970). If the duration $\Delta t$ of the trailing edge of the pulse is expressed in the form $\Delta t \propto \lambda^{\alpha}$, where $\lambda$ is wavelength, $\alpha$ is found to approach the value 4 asymptotically at the longer wavelengths. The effect was attributed to scattering from electron density inhomogeneities in the interstellar medium, most probably associated with the Gum Nebula.

Staelin and Sutton (1970) and Rankin et al. (unpublished) have found that for NP 0532 the pulse duration also increases rapidly at the longer wavelengths.

In May 1970 a second series of observations of PSR 0833-45 was undertaken at Parkes at a number of wavelengths between 20 and $100 \mathrm{~cm}$. In addition to the instantaneous flux density, the linearly polarized component and its position angle were measured and averaged over several thousand pulses.

These later results provide further support for the scattering hypothesis. They also support the view that the intrinsic pulse shape and instantaneous position angle are substantially independent of wavelength when propagation effects have been eliminated. The latter result agrees with that of Radhakrishnan and Cooke (1969) and Radhakrishnan (1969) derived from observations at much shorter wavelengths; it therefore supports their conclusion that the radiation originates from the vicinity of a magnetic pole, and well within the velocity of light cylinder.

\section{Equipment}

The observing technique has been described by Ables et al. (1970). It involved several 
receivers, each with its own linearly polarized feed. The receiver outputs were fed through a rapid multiplexer to a PDP-9 computer, which also received a pulse train at the Doppler-shifted pulsar period. The whole feed assembly could be rotated in position angle.

\section{Results}

The results for four of the observed wavelengths are shown in Figures 1-4. The observed values of flux density are indicated by filled circles and the position angle by open circles. Except for Figure 1 the full lines are not lines of best fit to the data

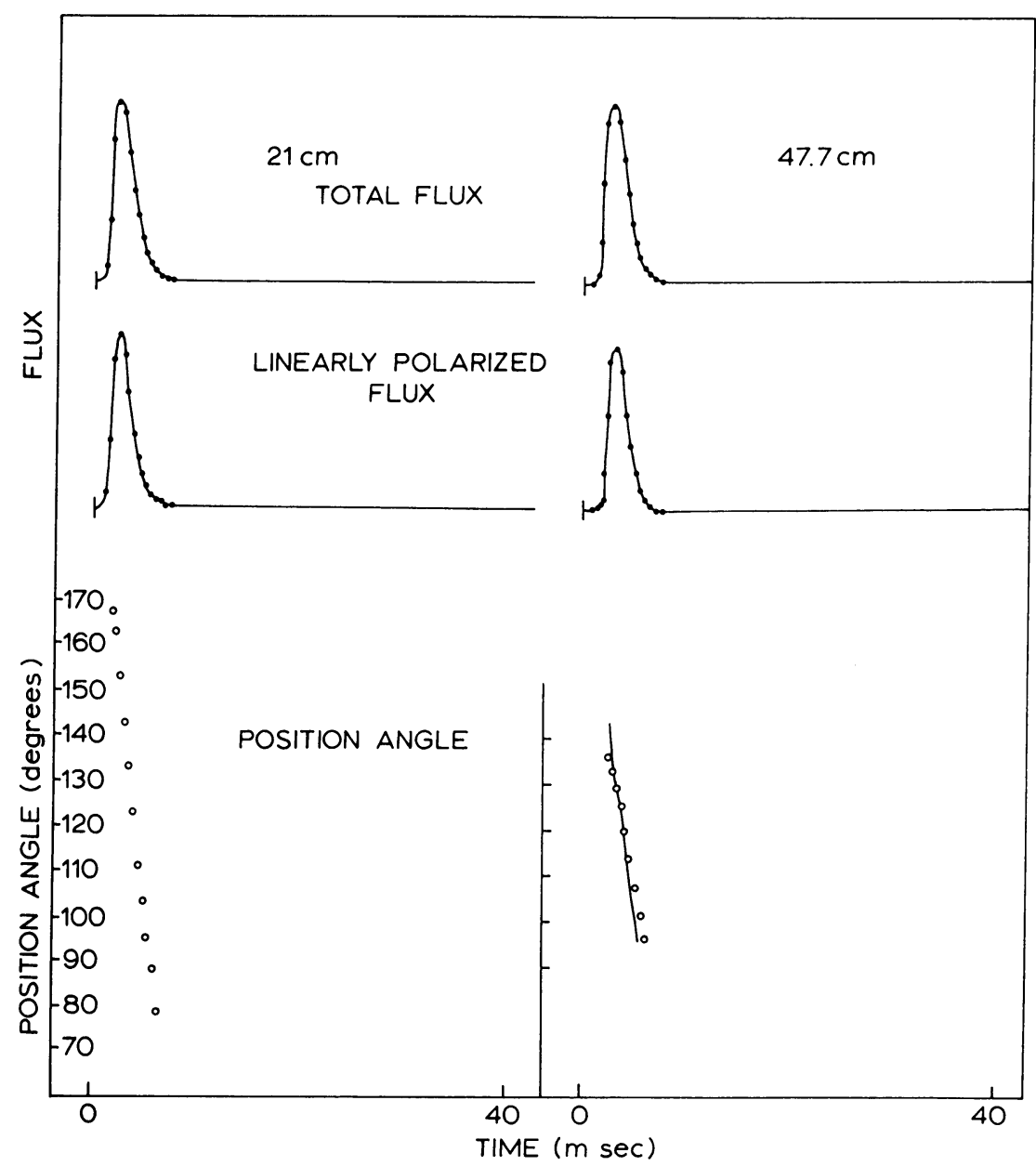

Fig. 1. Observed pulse shape and polarization at $21 \mathrm{~cm}$ wavelength. The filled circles represent the observed values of total flux density and the polarized component. The continuous lines represent the curves of best fit to the data points. Instantaneous position angles are indicated by open circles. The period is $89.2 \mathrm{msec}$.

Fig. 2. Observational results at $47.7 \mathrm{~cm}$. The filled and open circles have the same significance as for Figure 1, but the continuous lines have been computed by the convolution process described in the text. 


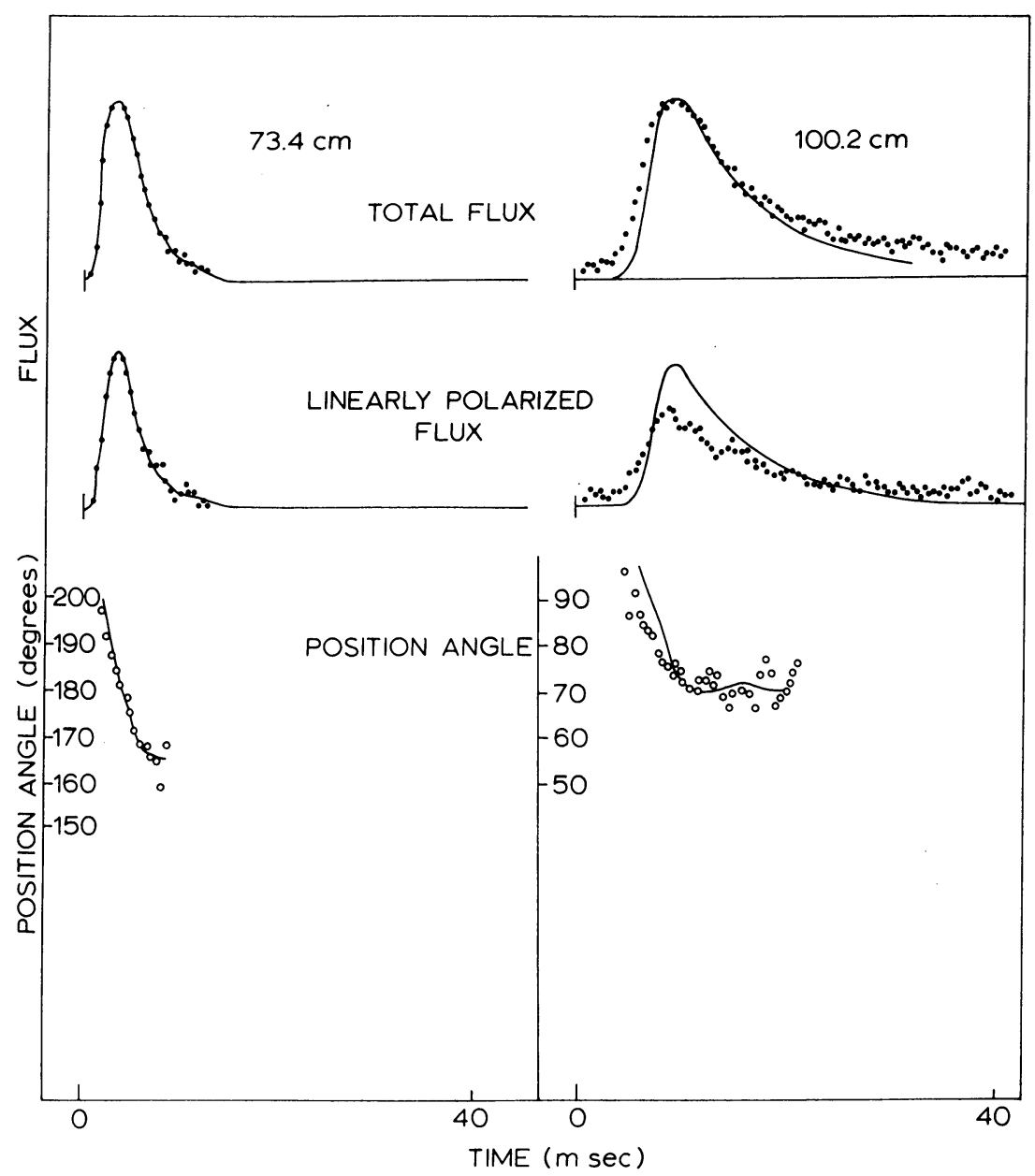

Fig. 3. Observational results at $73.4 \mathrm{~cm}$. The same convention has been used as in Figure 2 .

Fig. 4. Observational results at $100.2 \mathrm{~cm}$. The same convention has been used as in Figures 2 and 3 .

points, but are curves computed as indicated below. As pointed out previously, the pulse broadens with increasing wavelength. In addition, both the degree of linear polarization at the peak and the change of position angle within the pulse decrease as the wavelength increases.

\section{Interpretation}

The case of a plane wave traversing a 'thick' medium consisting of a larger number of randomly spaced irregularities along the line of sight has been considered by a number of authors. Fejer (1954), for example, has shown that the angular spectrum of the scattered component of the emergent radiation is approximately a twodimensional Gaussian function. For a radio wave traversing a very tenuous irregular 
ionized medium, the angular spread of the emergent wave varies as $\lambda^{2}$. According to arguments of the type adduced by Scheuer (1968), it then follows that a very short-duration pulse of radiation from a point source will show an approximately exponential decay after passing through such a scattering medium; this is the result of multipath transmission.

The effect of scattering on a pulse of finite duration may be computed by convolving the emitted pulse with the appropriate exponential function. If the scale of the

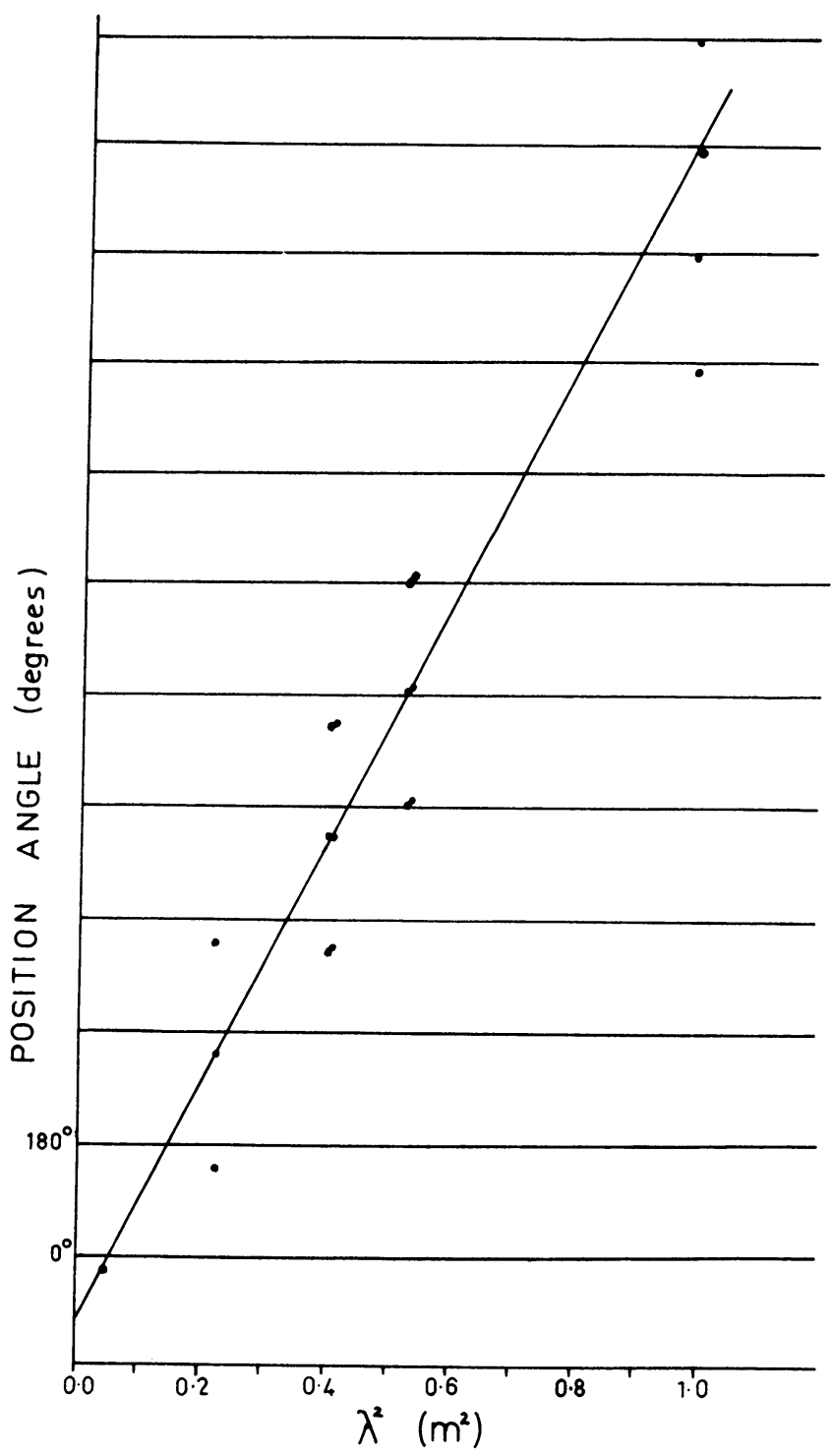

Fig. 5. Position angle of polarization at pulse peak vs. $\lambda^{2}$. The slope of the line which passes through all points corresponds to a rotation measure of $33.2 \mathrm{rad} \mathrm{m}^{-2}$. 
irregularities is much larger than the observing wavelength, and if magnetic field effects may be neglected, the effect of scattering is independent of polarization. It follows that the characteristics of the scattered pulse, including its instantaneous polarization, may be derived by convolving each Stokes parameter of the emitted pulse with the same exponential function; the scale of this function will of course be proportional to $\lambda^{4}$.

From the results of Radhakrishnan and Cooke (1969) we conclude that scattering has negligible effect on the pulse from PSR 0833-45 at wavelengths of $20 \mathrm{~cm}$ and shorter. We have therefore derived the scale of the exponential convolving function at $\lambda=100 \mathrm{~cm}$ by trial, by comparing the total flux pulse at this wavelength with that at $20 \mathrm{~cm}$. For the other wavelengths we have taken the scale width of the exponential to be proportional to $\lambda^{4}$.

\section{Discussion}

Following are the results of carrying out the convolutions of the assumed intrinsic pulse as described above:

(i) In all cases the calculated variation of polarization position angle agreed well with that observed (Figures 1(c) to 4(c)).

(ii) At $100 \mathrm{~cm}$ the predicted magnitude of the linearly polarized component of the pulse exceeded that observed by about $30 \%$.

(iii) For wavelengths of $73 \mathrm{~cm}$ and shorter no significant difference was found between the predicted and observed total intensity at any instant during the pulse. The same was true of the polarized intensity (Figures $2 b, 3 b$ ).

Finally, after making slight adjustments to the position angle at the pulse peak, which the convolution results indicated were necessary, it was found that the rotation measure was constant at $33.2 \mathrm{rad} \mathrm{m}^{-2}$ across the 5 to 1 wavelength range. The measured position angles are given in Figure 5. Taken in conjunction with the foregoing results this strongly indicates that the rotation of the position angle at the peak with wavelength is a propagation effect in the interstellar medium, and that the intrinsic position angle at any instant is independent of wavelength.

\section{Acknowledgements}

We would like to thank Mr. D. Smart for his assistance with the considerable amount of computer reduction involved, and Mrs. R. Raison for help with other aspects of the data reduction.

\section{References}

Ables, J. G., Komesaroff, M. M., and Hamilton, P. A.: 1970, Astrophys. Letters 6, 147.

Fejer, J. A.: 1954, Proc. Roy. Soc. London A220, 455.

Radhakrishnan, V.: 1969, Proc. Astron. Soc. Australia 1, 254.

Radhakrishnan, V. and Cooke, D. J.: 1969, Astrophys. Letters 3, 225.

Scheuer, P. A. G.: 1968, Nature 218, 920.

Staelin, D. H. and Sutton, J. M.: 1970, Nature 226, 69. 